\title{
CLAY INCOPORATION AT THE DIELECTRIC LAYER OF MULTILAYER POLYMER FILMS FOR ELECTROCHEMICAL ACTIVATION
}

Damia Amoura, ${ }^{1}$ Margarita Sánchez-Jiménez, ${ }^{2}$ Francesc Estrany,,${ }^{2,3}$ Laid Makhloufi, ${ }^{1}$ and Carlos Alemán ${ }^{3,4, *}$

${ }^{1}$ Laboratoire d'électrochimie, corrosion et de valorisation énergétique (LECVE),

Département de Génie des Procédés, A. Mira University, Route de TargaOuzemmour, 06000 Bejaia, Algeria

${ }^{2}$ Departament d'Enginyeria Química, Escola Universitaria d'Enginyeria Tècnica Industrial de Barcelona, Universitat Politècnica de Catalunya, Comte d'Urgell 187, 08036 Barcelona, Spain

${ }^{3}$ Center for Research in Nano-Engineering, Universitat Politècnica de Catalunya, Campus Sud, Edifici C', C/Pasqual i Vila s/n, Barcelona E-08028, Spain

${ }^{4}$ Departament d'Enginyeria Química, E. T. S. d'Enginyers Industrials, Universitat Politècnica de Catalunya, Diagonal 647, 08028, Barcelona, Spain

* francesc.estrany@upc.edu and carlos.aleman@upc.edu 


\begin{abstract}
The electrochemical properties (i.e. electroactivity, electrostability and specific capacitance) of 3-layered films made of poly(3,4-ethylenedioxythiophene) (PEDOT) and poly(N-methylpyrrole) (PNMPy) arranged alternatively have been activated by incorporating exfoliated montmorillonite (MMT) at the intermediate layer. 3-Layered films were prepared using the layer-by-layer electropolymerization technique, whereas the previously exfoliated clay was incorporated into PNMPy layer using an in situ polymerization procedure. The influence of the clay on the properties has been investigated as function of the thickness of the internal and external PEDOT layers, which has been controlled through the polymerization time. Micrometric PEDOT layers have been found to exert a template effect on the topography and morphology of the intermediate PNMPy or PNMPy-MMT layer, this effect being practically inexistent for films with nanometric PEDOT layers. The ability to exchange charge reversibly (electroactivity) and the electrochemical stability (electrostability) of films with MMT at the intermediate layer are higher than those of films without clay, these effects being more pronounced for films with micrometric PEDOT layers. The influence of the thickness is even more remarkable in the specific capacitance, which decreases rapidly with increasing thickness. The clay at the intermediate layer improves the specific capacitance of micrometric films and, most importantly, protects the capacitive behavior against electrochemical degradation in all cases. In summary, the presence of MMT in the intermediate PNMPy layer makes 3-layered films sensitive to the dielectric breakage, activating their electrochemical and capacitive properties. Such clay-induced effects are more pronounced for films made of micrometric PEDOT layers.
\end{abstract}

Keywords: Conducting polymers; Montmorillonite; PEDOT; Polypyrrole; Supercapacitor. 


\section{INTRODUCTION}

Conducting polymers (CPs) have recently attracted considerable scientific and technological interest because of their interesting electrical, electrochemical, optical and magnetic properties. ${ }^{1-4}$ Within this context, the high ability of CPs to store charge (i.e. electrical energy) deserves special consideration since such electrochemical activity (electroactivity) plays an important role in electronic devices, such as capacitors and supercapacitors. The latter are electrochemical capacitors that can be used as charge storage devices to provide higher power density, energy density, and longer life cycle than batteries and conventional capacitors. ${ }^{5-9}$ Therefore, improvement of electrode materials to achieve supercapacitors with better electrochemical properties is a challenge.

Poly(3,4-ethylenedioxythiophene), hereafter abbreviated PEDOT, is one of the most important CPs due to its high conductivity (up to $500 \mathrm{~S} / \mathrm{cm}$ for as prepared films and up to 2000-4000 S/cm after treatment with acids), good thermal and chemical stability, fast dopingdedoping processes, and excellent biocompatibility. ${ }^{10-16}$ Moreover, PEDOT presents excellent electrochemical behavior in terms of electroactivity and electrostability. ${ }^{17,18}$ In recent years, significant efforts have been devoted to improve the intrinsic capacitive properties of PEDOT. ${ }^{11,19}$ Within this context, composites based on transition-metal oxides (e.g. $\mathrm{MnO}_{2}$, $\mathrm{NiO}, \mathrm{Fe}_{3} \mathrm{O}_{4}$ and $\mathrm{V}_{2} \mathrm{O}_{5}$ ) embedded into the PEDOT matrix showed better capacitive properties than pure PEDOT. ${ }^{20-23}$ Other approaches were based on the preparation of nanocomposites of PEDOT and nanotubes. ${ }^{24-26}$

A few years ago we used the layer-by-layer ( $\mathrm{LbL}$ ) electrodeposition technique to prepare multilayered conducting systems based on PEDOT and poly(N-methylpyrrole) (PNMPy) layers arranged alternatively, ${ }^{27,28}$ hereafter abbreviated ml-PEDOT/PNMPy. These multilayered materials showed better electrochemical properties and a higher ability to store charge than each of the two individual CPs. Moreover, such improvement increased with the 
number of layers, which was attributed to a synergistic effect produced by the favorable interaction between the PEDOT and PNMPy layers at the corresponding interfaces. Thus, the coupling between PEDOT and PNMPy was found to be, in terms of enhancement of the electrochemical properties, significantly more positive in multilayered films than the interaction between the two monomers in the corresponding copolymers. ${ }^{29}$ In addition, the specific capacitance, $\mathrm{SC}$, of ml-PEDOT/PNMPy $(\mathrm{SC}=90 \mathrm{~F} / \mathrm{g}$ ) was found to be significantly higher than that of pure PEDOT $(\mathrm{SC}=41 \mathrm{~F} / \mathrm{g}$ ), even though both were lower than those reported for PEDOT-inorganic hybrid nanocomposites. ${ }^{8,30}$

The development of CP-clay nanocomposites is a field of increasing interest due to the important technological applications of these materials. ${ }^{9,31-35}$ Some hybrid PEDOT-clay nanocomposites based on montmorillonite (MMT), which is a smectite group mineral clay that belongs to the general family of 2:1 layered silicates, have been reported in the last few years. ${ }^{9,33,36-38}$ Since exfoliated CP-MMT nanocomposites usually have better properties (e.g. stiffness, strength and barrier property) than the intercalative ones, it is rationalized that the higher the degree of exfoliation, the greater the enhancement of these properties. ${ }^{39}$ Unfortunately, PEDOT-MMT nanocomposites prepared by Letaïef et al. ${ }^{33}$ and Rajapakse et $a l .{ }^{36}$ showed an intercalative structure rather than the more desirable exfoliated organization. Han and $\mathrm{Lu}^{37}$ reported exfoliated PEDOT-MMT nanocomposites that were obtained by in situ polymerization in aqueous media using modified clays. Although the thermal stability of such nanocomposites was better than that of pristine PEDOT, their electrical conductivity was very low (i.e. from $10^{-7}$ to $10^{-2} \mathrm{~S} / \mathrm{cm}$ ). ${ }^{37}$ More recently, some of us reported an in situ electropolymerization procedure to prepare exfoliated PEDOT-MMT nanocomposites using non-modified clays, the electrical conductivity of the produced materials ranging from 1 to 4 $\mathrm{S} \mathrm{cm}{ }^{-1} \cdot{ }^{37}$ Differences in the electrical conductivity of such two classes of PEDOT-MMT nanocomposites were attributed to both the efficiency of the dopant agent (i.e. the oxidant 
power of $\mathrm{LiClO}_{4}$ is higher than that of the organic dopant agents used in ref. 37) and the length of PEDOT chains, which is larger when prepared by anodic polymerization. Also, we extended our in situ polymerization procedure to the preparation of PEDOT-MMT nanocomposites with relatively high clay concentrations (i.e. of even $50 \% \mathrm{w} / \mathrm{w}$-dry weightin the monomer solution). ${ }^{9}$ The specific capacitance of supercapacitors prepared using an asymmetric PEDOT/PEDOT-MMT configuration $(\mathrm{SC}=116 \mathrm{~F} / \mathrm{g})$ was found to be $40 \%$ higher than that obtained for the symmetric PEDOT/PEDOT configuration $(\mathrm{SC}=82 \mathrm{~F} / \mathrm{g}) .{ }^{9}$

In this work, we explore the structural, electrochemical and capacitive properties of 3layered films made of two layers of PEDOT and an intermediate layer of PNMPy-MMT. The properties of this clay-containing multilayered system, hereafter denoted PEDOT/PNMPyMMT/PEDOT have been compared with those of the corresponding free-clay system, denoted PEDOT/PNMPy/PEDOT. The influence of the thickness, which has been controlled through the polymerization time $(\theta)$, on the clay-containing and clay-free 3-layered systems has been also investigated. Structure-property relationships have been established using morphological and topographical analyses based on scanning electron microscopy (SEM) and tapping-mode atomic force microscopy (AFM).

\section{METHODS}

Materials. 3,4-ethylenedioxythiophene (EDOT), N-methylpyrrole (NMPy) and acetonitrile were purchased from Sigma-Aldrich (Spain) and used as received. Montmorillonite (MMT) was purchased from Sigma-Aldrich (Spain) and used after sonication as described later. Anhydrous lithium perchlorate $\left(\mathrm{LiClO}_{4}\right)$ was purchased from Sigma-Aldrich (Spain) and was stored in an oven at $80{ }^{\circ} \mathrm{C}$ before use in the electrochemical trials. All chemicals were analytical reagent grade. 
Synthesis of PEDOT films and layers. PEDOT films were prepared by chronoamperometry (CA) under a constant potential of $1.40 \mathrm{~V}$ using a three-electrode onecompartment cell under nitrogen atmosphere (99.995\% in purity) at $25{ }^{\circ} \mathrm{C}$. Steel AISI 316 sheets of $2 \times 2 \mathrm{~cm}^{2}$ area were employed as working and counter electrodes. To avoid interferences during the electrochemical analyses, before each trial the working and counter electrodes were cleaned with ethanol, after that with acetone, and dried in an air-flow. The reference electrode was an $\mathrm{Ag} \mid \mathrm{AgCl}$ electrode containing a $\mathrm{KCl}$ saturated aqueous solution $\left(\mathrm{E}^{\mathrm{o}}=0.222 \mathrm{~V}\right.$ vs. standard hydrogen electrode at $\left.25^{\circ} \mathrm{C}\right)$. All the potentials reported in this work are referred to the $\mathrm{Ag} \mid \mathrm{AgCl}$ electrode. The cell was filled with $50 \mathrm{~mL}$ of $10 \mathrm{mM}$ EDOT monomer solution in acetonitrile containing $0.1 \mathrm{M} \mathrm{LiClO}_{4}$ as doping electrolyte. In order to evaluate the influence of the thickness on the electrochemical properties, PEDOT films were generated using the following polymerization times $\theta_{P E D O T}=10,20,30,40,50,100$ and 300 s. All electrochemical experiments were conducted on a PGSTAT101 AUTOLAB potentiostat-galvanostat connected to a PC computer and controlled through the NOVA 1.6 software.

Synthesis of PNMPy(MMT) and PNMPy films and layers. The amount of MMT in the monomer solution used to prepare PNMPy(MMT) was 100\% w/w (dry weight referred to the NMPy monomer concentration). Exfoliation of MMT in de-ionized water was performed by sonication. ${ }^{9,38}$ For this purpose, an ultrasonic homogenizer BANDELIN sonopuls HD 2200 was used to sonicate MMT in de-ionized water (neutral $\mathrm{pH}$ ) for 20 minutes by applying successive cycles of 1 minute of sonication at $15 \%$ of power. ${ }^{38}$ The resulting solution was stirred for 3 hours in a frozen environment (ice) using a magnetic stirrer at $500 \mathrm{rpm}$. After this, a $10 \mathrm{mM}$ NMPy solution in deionized water with $0.1 \mathrm{M} \mathrm{LiClO}_{4}$ was added to the exfoliated clay solution, and stirred for $5 \mathrm{~min}$. PNMPy(MMT) was deposited by CA using a constant potential of $1.10 \mathrm{~V}$. Three polymerization times were used in this work: $\theta_{P N M P y}=$ 
180, 300 and 600 s. Films of PNMPy were prepared using identical conditions but without adding MMT to the generation medium.

Synthesis of 3-layered films. The electrochemical LbL technique was used to prepare the PEDOT/PNMPy-MMT/PEDOT and PEDOT/PNMPy/PEDOT films. For the generation of each layer the working electrode was immersed for given polymerization time in the cell with the corresponding generation solution. Accordingly, the total polymerization time $(\tau)$ was $\tau=$ $2 \cdot \theta_{P E D O T}+\theta_{P N M P y}$. It should be noted that the external and internal layers of all 3-layered films were made of PEDOT, PNMPy-MMT or PNMPy being always located at the intermediate layer. The experimental conditions used to produce PEDOT and PNMPy-MMT or PNMPy layers correspond to those described above. Figure 1 illustrates the synthetic procedure used to produce PEDOT-PNMPy(MMT)-PEDOT films. The main novelties of the multilayered systems studied in this work involve not only the incorporation of clay at the intermediate layer but also the combination of layers prepared using different reaction media (i.e. organic solution for PEDOT layers and water for PNMPy-MMT and PNMPy).

Scanning electron microscopy (SEM). SEM studies were performed to examine the surface morphology. Dried samples were placed in a Focused Ion Beam Zeiss Neon 40 scanning electron microscope operating at $3 \mathrm{kV}$, equipped with an EDX spectroscopy system. Samples were mounted on a double-side adhesive carbon disc and sputter-coated with a thin layer of carbon to prevent sample charging problems.

Atomic Force Microscopy (AFM). Topographic AFM images were obtained with a Dimension 3100 Nanoman AFM and Multimode from Veeco using a Nanoscope IV controller under ambient conditions in tapping mode. The root-mean-square roughness was determined using the statistical application of the Nanoscope software, which calculates the average considering all the values recorded in the topographic image with exception of the maximum and the minimum. AFM measurements were performed on various parts of the 
films, which produced reproducible images similar to those displayed in this work. The scan window size was $5 \times 5 \mu \mathrm{m}^{2}$.

\section{Electrochemical properties: Cyclic voltammetry (CV) and chronopotentiometry (CP).}

The electrochemical properties of the studied systems were determined by CV using acetonitrile solution with $0.1 \mathrm{M} \mathrm{LiClO}_{4}$. The initial and final potentials were $-0.5 \mathrm{~V}$, and the reversal potential was $1.6 \mathrm{~V}$. A scan rate of $100 \mathrm{mV} \cdot \mathrm{s}^{-1}$ was used. The ability to exchange charge reversibly (i.e. electroactivity) and the electrochemical stability (i.e. electrostability) were determined through direct measure of the anodic and cathodic areas in the control voltammograms using NOVA software. The loss of electroactivity (LEA, in \%) was expressed as:

$$
\mathrm{LEA}=\frac{\Delta \mathrm{Q}}{\mathrm{Q}_{\mathrm{II}}} \cdot 100
$$

where $\Delta \mathrm{Q}$ is the difference of voltammetric charges (in $\mathrm{C}$ ) between the second and the last cycle, and $\mathrm{Q}_{\mathrm{II}}$ is the voltammetric charge corresponding to the second cycle.

The specific capacitance (in F.g ${ }^{-1}$ ) of the active materials in the electrodes was calculated as:

$$
\mathrm{S} C=\frac{\mathrm{Q}}{\Delta \mathrm{Vm}}
$$

where $\mathrm{Q}$ is the voltammetric charge, which is determined by integrating either the oxidative or the reductive parts of the cyclic voltammogram curve, $\Delta \mathrm{V}$ is the potential window and $\mathrm{m}$ is the mass of polymer on the surface of the working electrode. The latter is derived from the productivity current and polymerization charge. ${ }^{18}$

A current of $2 \mathrm{~mA}$ was applied by $\mathrm{CP}$ for $900 \mathrm{~s}$ to determine the potential of the maximum resistance of the three-layered system in acetonitrile solution with $0.1 \mathrm{M} \mathrm{LiClO}_{4}$.

The thickness of the films was estimated from the mass of polymer deposited in the electrode. The latter procedure was detailed in previous works. ${ }^{27,28}$ PEDOT and PNMPy 
densities were taken from previous studies based on identical experimental conditions (i.e. the same potential, solvent and concentration of electrolyte). ${ }^{18,29,40}$

\section{RESULTS AND DISCUSSION}

Polymerization time and thickness. In order to determine the minimum value of $\theta_{P N M P y}$ required for the preparation of homogeneous intermediate PNMPy or PNMPy-MMT layers in 3-layered systems, preliminary assays on 2-layered films were carried out. The exfoliation of clays in the PNMPy-MMT layer was successful in all cases, as is illustrated in the TEM micrograph displayed in Figure 2. It should be noted that, due to the incorporation of the exfoliated clay, the experimental conditions used in this work to generate the layer based on PNMPy are different from those previously reported for multilayered films (i.e. $10 \mathrm{mM}$

NMPy solution in acetonitrile with $0.1 \mathrm{M} \mathrm{LiClO}_{4}$ at a constant potential of $1.40 \mathrm{~V}^{8,27,286,41}$ ). Initially, 2-layered systems were prepared by depositing a PNMPy or PNMPy-MMT layer generated using $\theta_{P N M P Y}=180 \mathrm{~s}$ onto a PEDOT layer that was previously deposited onto a steel electrode using $\theta_{P E D O T}=300 \mathrm{~s}$. AFM images of such PEDOT/PNMPy and PEDOT/PNMPyMMT films are displayed in Figures $3 a$ and $3 b$, respectively. As it can be seen, the triangular overlapping edges found for the two 2-layered films largely resemble the topography obtained for the underlying PEDOT layer (Figure 4a).,42

On the other hand, electrochemical studies on 2-layered films prepared using $\theta_{P E D O T}=300$ $\mathrm{s}$ and $\theta_{P N M P y}=180 \mathrm{~s}$ suggested an incomplete covering of the internal PEDOT layer by PNMPy or PNMPy-MMT. Thus, the electroactivities determined for different samples of each 2-layered system were found to be very diverse and non-reproducible. This situation is completely different from that found for 2-layered films in which the central PNMPy layer was prepared in acetonitrile solution at a constant potential of $1.40 \mathrm{~V} .^{8,27,28,41}$ In such case, $\theta_{P N M P y}$ values ranging from 10 to $100 \mathrm{~s}$ were evaluated, fully reproducible cyclic 
voltammograms being obtained for all them. The deficient coverage of the internal PEDOT film by the PNMPy or PNMPy-MMT layer prepared in water at $1.10 \mathrm{~V}$ precludes the correct dielectric effect of the intermediate layer, which is less conductive than the PEDOT layers. Accordingly, preferential paths of electric current are formed, distorting the results and introducing randomness, which explain the lack of reproducibility of the cyclic voltammograms.

In order to overcome such limitations, $\theta_{P N M P y}$ was increased from $180 \mathrm{~s}$ to 300 and $600 \mathrm{~s}$. Results derived from 2-layered films prepared using $\theta_{P E D O T}=\theta_{P N M P y}=300 \mathrm{~s}$ (not shown) reflected the deficiencies mentioned above. AFM height images of 2-layered films prepared using $\theta_{P E D O T}=300 \mathrm{~s}$ and $\theta_{P N M P y}=600 \mathrm{~s}$ are displayed in Figures $3 \mathrm{c}$ and $3 \mathrm{~d}$. It is worth nothing that the surface topographies of both PNMPy and PNMPy-MMT layers also resemble the topography previously reported for individual PEDOT, ${ }^{11,42}$ as occurred above for $\theta_{P N M P y}=180$ s. However, cyclic voltammograms recorded for PEDOT/PNMPy and PEDOT/PNMPy-MMT films prepared using $\theta_{P E D O T}=300 \mathrm{~s}$ and $\theta_{P N M P y}=600 \mathrm{~s}$ (not shown) were fully reproducible. The thickness of the PEDOT layer prepared using $\theta_{P E D O T}=300 \mathrm{~s}$ was found to be $1.1 \pm 0.1 \mu \mathrm{m}$, whereas those of PNMPy and PNMPy-MMT layers obtained using $\theta_{P N M P y}=600 \mathrm{~s}$ was $1.8 \pm 0.1$ $\mu \mathrm{m}$. According to these results, PNMPy and PNMPy-MMT prepared using $\theta_{P N M P Y}=600 \mathrm{~s}$ completely cover the internal PEDOT layer, which affects the topography of the formers acting as a structural template (see next sub-section).

\section{Thickness and template effect of the internal PEDOT layer}

Once the polymerization time required for the intermediate PNMPy or PNMPy-MMT layer has been determined, the thickness of the PEDOT layer has been modulated to consider different surface topographies and morphologies. Thus, it is expected that the surface roughness, which in PEDOT increases with the thickness of the film, ${ }^{8,30,41}$ affects the 
topography and morphology of PNMPy or PNMPy-MMT layer. Accordingly, in addition of the $\theta_{P E D O T}=300 \mathrm{~s}$ employed in the previous sub-section, polymerization times for internal PEDOT layer of 10, 20, 30, 40, 50 and $100 \mathrm{~s}$ were also considered. Figures $3 \mathrm{e}$ and $3 \mathrm{f}$ display the topographic AFM images of PNMPy and PNMPy-MMT layers, respectively, of 2-layered systems prepared using $\theta_{P E D O T}=50 \mathrm{~s}$ and $\theta_{P N M P Y}=600 \mathrm{~s}$, while the AFM image of the underlying PEDOT layer is provided in Figure 4b. As it can be seen, the resulting globular morphology, which is characteristic of PNMPy films directly deposited on steel, ${ }^{42}$ is completely different from those of 2-layered systems obtained using $\theta_{\text {PEDOT }}=300 \mathrm{~s}$ (Figures 3a-3d). These observations suggest that the role of the internal PEDOT layer as structural template of the PNMPy or PNMPy-MMT layer is practically inexistent for $\theta_{\text {PEDOT }} \leq 50 \mathrm{~s}$. This assumption was corroborated through the AFM images (not shown) of 2-layered films prepared using $\theta_{P E D O T}=10 \mathrm{~s}$ and $\theta_{P N M P y}=600 \mathrm{~s}$. The thickness of PEDOT layers obtained using $\theta_{P E D O T}=10$ and $50 \mathrm{~s}$ were 139 and $271 \mathrm{~nm}$, respectively.

SEM micrographs of PNMPy and PNMPy-MMT films $\left(\theta_{P N M P y}=600 \mathrm{~s}\right)$ deposited onto an internal PEDOT layer of nanometric thickness $\left(\theta_{P E D O T}=10 \mathrm{~s}\right)$ are displayed in Figure 5a and $5 b$, respectively. As it can be seen, the internal PEDOT layer is completely coated in both cases, corroborating previous AFM observations. Furthermore, PNMPy and PNMPy-MMT layers present relatively compact globular morphologies similar to that reported for PNMPy films directly electrodeposited onto a steel substrate. ${ }^{8}$ Accordingly, the template effect of the internal PEDOT layer is practically inexistent. Despite the similarity between PNMPy and PNMPy-MMT morphologies, it should be noted that the diameter of the globules is around $30 \%$ larger for the latter than for the former.

The morphology of PNMPy and PNMPy-MMT layers, $\theta_{P N M P Y}=600 \mathrm{~s}$, deposited onto micrometric PEDOT layers obtained using $\theta_{P E D O T}=300 \mathrm{~s}$ is displayed in Figure $5 \mathrm{c}$ and $5 \mathrm{~d}$, respectively, while the surface morphology of the internal PEDOT layer is provided in Figure 
5e. As it can be seen, the surface morphologies are completely different to those discussed above for films deposited onto nanometric PEDOT (Figures 5a and 5b), evidencing the predominant structural role of the internal PEDOT layer, which acts as a soft template. SEM micrograph displayed in Figure 5c reflects a heterogeneous and porous morphology formed by micrometric blocks of grouped nanosheet-like structures. This morphology largely resembles that previously reported for micrometric PEDOT films (Figure 5e) and, therefore, can be simply interpreted as an ultra-thin PNMPy coating adapted to the structure of the latter. Comparison between Figures 5c and 5d evidences some morphological differences between PNMPy and PNMPy-MMT deposited onto micrometric PEDOT, which have been ascribed to the incorporated clay. More specifically, MMT provokes an increment in the size of the nanosheet-like structures, this effect being similar to that described above for PNMPy-MMT deposited onto nanometric PEDOT. Despite of the influence of the clay on the morphology, the porosity and heterogeneity of the internal template is reflected in both Figures $5 \mathrm{c}$ and $5 \mathrm{~d}$.

According to these results, which are independent of the presence of clay in the intermediate layer, in the next sub-sections we have determined the influence of the template effect induced by the PEDOT layer in the electrochemical and capacitive properties of PEDOT/PNMPy/PEDOT and PEDOT/PNMPy-MMT/PEDOT films. For this purpose, 3layered films involved PEDOT layers of both nanometric ( $\theta_{P E D O T}$ ranging from 10 to $50 \mathrm{~s}$ ) and micrometric $\left(\theta_{\text {PEDOT }}=100\right.$ and $\left.300 \mathrm{~s}\right)$ thickness were considered.

\section{Electrochemical activity and stability of 3-layered films}

The variations of the electroactivity and electrochemical stability of 3-layered films as a function of the thickness of PEDOT layers were evaluated considering $\theta_{P N M P Y}=600 \mathrm{~s}$ in all cases. Figure 6 represents the first control voltammogram of 3-layered films prepared using $\theta_{\text {PEDOT }}=300 \mathrm{~s}$. The electroactivity, which increases with the similarity of the cathodic and 
anodic areas, is $22 \%$ higher for PEDOT/PNMPy-MMT/PEDOT (Figure 6a) than for PEDOT/PNMPy/PEDOT (Figure 6b), indicating that the clay facilitates the charge storage. Furthermore, the voltammogram recorded for the MMT-containing 3-layered film shows the nearly rectangular shape characteristic of capacitor devices, which is much less evident in the film without clay at the intermediate layer. Voltammograms recorded for 3-layered films prepared using $\theta_{P E D O T}=50 \mathrm{~s}$, which are also displayed in Figure 6, indicate that the electroactivity PEDOT/PNMPy-MMT/PEDOT (Figure 6c) is only $3 \%$ higher than that of PEDOT/PNMPy/PEDOT (Figure 6d).

These results indicate that, although the ability to exchange charge reversibly of MMTcontaining 3-layered films is higher than that of films without MMT, differences are only appreciable for films with micrometric PEDOT layers. In general, analysis of the voltammograms recorded from independent experiments using different $\theta_{P E D O T}$ values corroborated this feature. However, in some punctual cases (e.g. some 3-layered films prepared using $\theta_{\text {PEDOT }}=10,40$ and $100 \mathrm{~s}$ ) the electroactivity of films without MMT was found to be slightly higher than that of the corresponding MMT-containing film, suggesting a different distribution of the clay in the intermediate layer or a different structure at the PEDOT/PNMPy-MMT interphase. In spite of this anomalous behavior, we observed that in all such cases the electroactivity of MMT-containing 3-layered films becomes higher than that of the films without clay after a few consecutive oxidation-reduction cycles. This feature is clearly reflected in Figure 7, which represents the evolution of the electroactivity difference between PEDOT/PNMPy-MMT/PEDOT and PEDOT/PNMPy/PEDOT prepared using $\theta_{P E D O T}=10,40$ and $100 \mathrm{~s}$. As it can be seen, in all cases the electroactivity of the latter film was slightly higher than that of the former at the beginning, even though this behavior was inverted after only 10 redox cycles. The overall of these observations allow us to state that 
PEDOT/PNMPy-MMT/PEDOT films are more electroactive that PEDOT/PNMPy/PEDOT films, independently of $\theta_{\text {PEDOT. }}$

The electrochemical stability was evaluated through the LEA, which is based on the variation of the oxidation and reduction areas of consecutive control voltammograms (Eqn 1). Thus, electrostability decreases with increasing LEA. Figure 8 compares the variation of the LEA against the number of redox cycles for PEDOT/PNMPy/PEDOT and PEDOT/PNMPyMMT/PEDOT prepared using $\theta_{P E D O T}$ values ranging from 10 to $300 \mathrm{~s}$ and $\theta_{P N M P y}=600 \mathrm{~s}$. As it can be seen, the LEA of the two 3-layered systems increases with decreasing thickness of the PEDOT layers. Accordingly, the electrochemical stability of 3-layered films with micrometric PEDOT layers is higher than that of films with nanometric PEDOT layers, independently of the presence of clay at the intermediate layer. Comparison between PEDOT/PNMPy/PEDOT and PEDOT/PNMPy-MMT/PEDOT films prepared using identical $\theta_{\text {PEDOT }}$ reveals that the clay provokes a significantly enhancement of the electrochemical stability for films made of both micrometric and nanometric PEDOT layers. For example, the LEA is $21 \%$ and $67 \%$ for MMT-containing 3-layered films obtained using $\theta_{P E D O T}=300$ and $10 \mathrm{~s}$, respectively, increasing to $40 \%$ and $82 \%$, respectively, for films without MMT at the intermediate layer. It should be noted that consecutive redox cycles provoke overoxidation and the formation of crosslinks at the PNMPy layer (i.e. PEDOT molecules are exclusively formed by $\alpha, \alpha$-linkages since the dioxane ring fused onto the thiophene ring occupies the $\beta$ positions of the latter), resulting in a significant physical degradation that close the structure. In PNMPy-MMT layers, the clay, which is not susceptible to overoxidation, acts as a physical support holding for more redox cycles an open structure.

In order to have a better characterization of the electrochemical stability of films made of micrometric and nanometric PEDOT layers, 3-layered films prepared using $\theta_{P E D O T}=10$ and $300 \mathrm{~s}$ were submitted to 500 consecutive redox cycles. The LEA calculated for 
PEDOT/PNMPy/PEDOT and PEDOT/PNMPy-MMT/PEDOT prepared using $\theta_{\text {PEDOT }}=300 \mathrm{~s}$ is $46 \%$ and $32 \%$, respectively. Comparison with results obtained for 100 redox cycles indicates that last 400 redox cycles provoked an electroactivity reduction of only $6 \%$ and $11 \%$, respectively. A similar behavior was observed for 3-layered films made of nanometric PEDOT layers, even though the electrochemical stability was considerably lower than those of films with micrometric PEDOT layers. Thus, the LEA of PEDOT/PNMPy/PEDOT and PEDOT/PNMPy-MMT/PEDOT prepared using $\theta_{P E D O T}=10 \mathrm{~s}$ was $92 \%$ and $82 \%$, respectively, after 500 redox cycles. Accordingly, the last 400 redox cycles induces an electroactivity reduction of $10 \%$ and $15 \%$, respectively. Despite this detriment is slightly larger for the MMT-containing film, it should be remarked that the electroactivity of the PEDOT/PNMPy-MMT/PEDOT film is $102 \%$ higher than that of PEDOT/PNMPy/PEDOT after 500 consecutive redox cycles.

\section{Capacitive behavior of 3-layered films}

Figure 9 represents the SC (Eqn 2) of PEDOT/PNMPy/PEDOT and PEDOT/PNMPy-

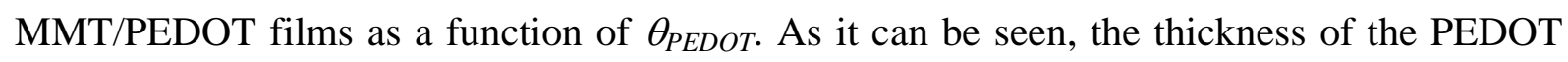
layers plays a crucial role in the capacitance of 3-layered films, the SC decreasing rapidly with increasing thickness. More specifically, the remarkably high SC of PEDOT/PNMPy/PEDOT decreases from $445 \mathrm{~F} \cdot \mathrm{g}^{-1}$ for $\theta_{\text {PEDOT }}=10 \mathrm{~s}$ to $54 \mathrm{~F} \cdot \mathrm{g}^{-1}$ for $\theta_{\text {PEDOT }}=$ 300 s. A similar behavior is observed for MMT-containing 3-layered films, the SC decreasing from 333 to $75 \mathrm{~F} \cdot \mathrm{g}^{-1}$ when $\theta_{\text {PEDOT }}$ increases from 10 to $300 \mathrm{~s}$. On the other hand, the relative influence of the clay on the SC has been found to depend on the thickness of PEDOT layers also. Thus, the SC of PEDOT/PNMPy/PEDOT is systematically higher than that of PEDOT/PNMPy-MMT/PEDOT for films with nanometric PEDOT layers ( $\theta_{\text {PEDOT }} \leq 50 \mathrm{~s}$ ). For films prepared $\theta_{P E D O T}=100 \mathrm{~s}$, the SCs of the two types of 3-layered systems differ in less than 
$10 \%$ (i.e. $\mathrm{SC}=98$ and $106 \mathrm{~F} \cdot \mathrm{g}^{-1}$ for films with and without MMT). Finally, the SC of PEDOT/PNMPy/PEDOT films obtained using $\theta_{P E D O T}=300 \mathrm{~s}$ is $\sim 40 \%$ lower than that of the corresponding 3-layered films with MMT at the intermediate layer.

The SCs of nanometric PEDOT/PNMPy/PEDOT and PEDOT/PNMPy-MMT/PEDOT films are also considerably larger than those previously reported for individual PEDOT and PEDOT-MMT films of similar thickness $(\sim 200 \mathrm{~nm}),{ }^{9}$ which were 350 and $250 \mathrm{~F} \cdot \mathrm{g}^{-1}$, respectively. However, differences between 3-layered and individual micrometric films are practically inexistent. Thus, the SC of PEDOT and PEDOT-MMT individual films prepared using $\theta_{\text {PEDOT }}=300 \mathrm{~s}$ is 56 and $96 \mathrm{~F} \cdot \mathrm{g}^{-1}$, respectively. ${ }^{9}$ The SC of PEDOT-inorganic hybrid nanocomposites involving $\mathrm{MoO}_{3}, \mathrm{RuO}_{2}$, carbon nanotubes, $\mathrm{MnO}_{2}$ and $\mathrm{NiFe}_{2} \mathrm{O}_{4}$ varies between 153 and $300 \mathrm{~F} \cdot \mathrm{g}^{-1} \cdot{ }^{21,44-49}$ These values evidence the enormous interest of nanometric 3-layered films as electrochemical capacitors.

In order to evaluate the influence of the electrochemical degradation in the capacitive behavior of 3-layered films, SC of PEDOT/PNMPy-MMT/PEDOT and PEDOT/PNMPy/PEDOT films prepared using $\theta_{\text {PEDOT }}=10,50$ and $300 \mathrm{~s}$ were measured after 100 consecutive oxidation-reduction cycles. Results are compared in Table 1 with those obtained for fresh samples. MMT offers a protection of the capacitive behavior against electrochemical degradation. Thus, the SC of 3-layered films without MMT at the intermediate layer obtained using $\theta_{P E D O T}=10,50$ and $300 \mathrm{~s}$ undergoes a reduction of $82 \%$, $63 \%$ and $37 \%$, respectively, after 100 redox cycles while the decrease is of only $69 \%, 41 \%$ and 23\% for films with PNMPy-MMT at the central layer.

\section{Chronoamperometric behavior of 3-layered films}

Figure 10 compares the chronopotentiograms recorded for 3-layered films prepared using $\theta_{\text {PEDOT }}=10,50$ and $100 \mathrm{~s}$. Results suggest that MMT reduces the resistance of the PNMPy 
dielectric layer. This effect is more pronounced with decreasing thickness of the PEDOT layers, which is consistent with the capacitive behavior displayed in the previous sub-section. The maximum potential for PEDOT/PNMPy/PEDOT films obtained using $\theta_{P E D O T}=10,50$ and $100 \mathrm{~s}$, which is reached after 136, 254 and $395 \mathrm{~s}$, respectively, is $1.91,1.84$ and $1.77 \mathrm{~V}$ (average values for three replicas). These values change to $1.66,1.77$ and $1.77 \mathrm{~V}$ for PEDOTPNMPy(MMT)-PEDOT films with $\theta_{P E D O T}=10,50$ and $100 \mathrm{~s}$, respectively, while the time required to reach such maximum potential evolves towards 102, 334 and 405 s, respectively. Accordingly, MMT provokes a reduction of $0.25 \mathrm{~V}$ in 3-layered films obtained using $\theta_{\text {PEDOT }}=$ $10 \mathrm{~s}$, indicating that the strength of binding between the adjacent PEDOT-PNMPy layers decreases in presence of the clay. This effect disappears upon increase the thickness of PEDOT layers. Thus, the difference between the maximum potentials measured for 3-layered films with and without MMT is of only 0.07 for $\theta_{P E D O T}=50 \mathrm{~s}$ and practically inexistent for and $\theta_{P E D O T}=100 \mathrm{~s}$. This behavior is fully consistent with the ability to exchange charge reversibly of 3-layered films discussed above.

The influence of the clay in the dielectric strength of the PNMPy layer becomes balanced by PEDOT layers when the thickness of the latter is micrometric. This effect can be explained by considering that, when the dielectric blocks the passage of electric current through the capacitor, charge is accumulated in the outer layers of PEDOT increasing the electrical potential between the plates of the capacitor. Accordingly, accumulated charge increases with the thickness of the outer PEDOT layer, which provokes a more gradual increment of the potential. These results allow us to conclude that the presence of MMT in the intermediate PNMPy layer makes the 3-layered system sensitive to the dielectric breakage when the potential increases instantaneously (i.e. nanometric PEDOT layers), such negative effect disappearing when the potential increase occurs slowly (i.e. micrometric PEDOT layers). 


\section{CONCLUSION}

The electrochemical and capacitive properties of PEDOT/PNMPy-MMT/PEDOT films have been compared with those of PEDOT/PNMPy/PEDOT. Independently of the presence of clay, the properties of such 3-layered systems have been found to depend on the thickness of PEDOT layers. Both AFM images and SEM micrographs clearly reflect the template effect exerted by micrometric PEDOT layers onto the topography and morphology of PNMPy or PNMPy-MMT intermediate layer. This template effect, which decreases with the thickness of the PEDOT layer, is practically inexistent for 3-layered films obtained using $\theta_{P E D O T}=10 \mathrm{~s}$ while it is very remarkable for films prepared using $\theta_{P E D O T}=300 \mathrm{~s}$. Thus, morphological changes associated to such template phenomenon have been found to be responsible of the differences between the properties of 3-layered films with different thickness. On the other hand, the minimum polymerization required to obtain a homogeneous and complete PNMPy or PNMPy-MMT layer time in aqueous solution is $\theta_{P N M P Y}=600 \mathrm{~s}$, electrochemical measures being not reproducible for 3-layered films prepared using $\theta_{P N M P y}<600 \mathrm{~s}$.

The electroactivity and electrochemical stability of films with micrometric PEDOT layers are higher than those of films with nanometric layers, independently of the incorporation of clay at the intermediate PNMPy layer. However, for a given thickness of PEDOT layers both the electroactivity and the electrochemical stability increase upon the incorporation of MMT at the intermediate layer. Also, the clay improves the resistance of the capacitive behavior of 3-layered films against electrochemical degradation. All these benefits, which increase with the thickness of the PEDOT layers, have been attributed to the dielectric breakage promoted by the intermediate layer. The overall of these results clearly indicate that MMT activates the electrochemical properties of PEDOT/PNMPy/PEDOT films, which represents a significant improvement, especially considering that the clay is electrochemically inactive. 


\section{ACKNOWLEDGEMENTS}

This work has been supported by MICINN and FEDER funds (project number MAT201234498).

\section{REFERENCES}

[1] Heinze J, Frontana-Uribe BA, Ludwigs S. Chem Rev 2010; 110: 4724-4771.

[2] Rubinson JF, Kayinamura YP. Chem Soc Rev 2009; 38: 3339-3347.

[3] Li C, Bai H, Shi G. Chem. Soc. Rev. 2009; 38: 2397-2409.

[4] Long YZ, Li MM, Gu CZ, Wan MX, Duvail JL, Liu ZW, Fan ZY. Prog Polym Sci 2011; 36: $1415-1442$.

[5] Kotz R, Carlen M. Electrochim Acta 2000; 45: 2483-2498.

[6] Frackowiak E, Beguin F. Carbon 2002; 40: 1775-1787.

[7] Wang Y, Zhang X. Electrochim Acta 2004; 49: 1957-1962.

[8] Aradilla D, Estrany F, Alemán C. J Phys Chem C 2011; 115: 8430-8438.

[9] Aradilla D, Azambuja D, Estrany F, Casas MT, Ferreira CA, Alemán C. J Mater Chem 2012; 22: 13110-13122.

[10] Lota K, Khomenko V, Frackowiak E. J Phys Chem Solids 2004; 65: 295-301.

[11] Ryu KS, Lee YG, Hong YS, Park YJ, Wu XL, Kim KM, Kang MG, Park NG, Chang SH. Electrochim Acta 2004; 50: 843-847.

[12] Ghosh S, Inganas O. Electrochem Solid State Lett 2000; 3: 213-215 .

[13] del Valle LJ, Estrany F, Armelin E, Oliver R, Alemán C. Macromol Biosci 2008; 8: 1144-1151.

[14] Fabregat G, Teixeira-Dias B, del Valle LJ, Armelin E, Estrany F, Alemán C. ACS Appl Mater Interfaces 2014; 6: 11940-11954. 
[15] Mengistie DA, Ibrahem MA, Wang P-C, Chi C-W. ACS Appl Mater Interfaces 2014; 6: 2292-2299.

[16] Kim N, Kee S, Lee S H, Lee BH, Kahng YH, Jo Y-R, Kim B-J, Lee K. Adv Mater 2014; 26: 2268-2272.

[17] Tamburri E, Orlanducci S, Toschi F, Terranova ML, Passeri D. Synth Met 2009; 159: 406-414.

[18] Ocampo C, Oliver R, Armelin E, Aleman C , Estrany F. J Polym Res 2006; 13: 193-200.

[19] Stenger-Smith D, Webber CK, Anderson N, Chafin AP, Zong K, Reynolds JR. J Electrochem Soc 2002; 149: A973-A977.

[20] Babakhani B, Ivey DG. Electrochim Acta 2010; 55: 4014-4024.

[21] Sen P, De A, Electrochim Acta 2010; 55: 4677-4684.

[22] Fusalba F, Ho HA, Breau L, Belanger D. Chem Mater 2000; 12: 2581-2589.

[23] Chen L, Yuan C, Dou H, Gao B, Chen S, Zhang X. Electrochim Acta 2009; 54: 23352341.

[24] Peng C, Snook GA, Fray DJ, Shaffer MSP, Chen GZ. Chem Commun 2006; 44: 46294631.

[25] Peng C, Jin J, Chen GZ, Electrochim Acta 2007; 53: 525-537.

[26] Rudge A, Davey J, Raistrick I, Gottesfeld S, Ferraris JP. J Power Sources 1994; 47: 89107.

[27] Estrany F, Aradilla D, Oliver R, Aleman C. Eur Polym J 2007; 43: 1876-1882.

[28] Estrany F, Aradilla D, Oliver R, Armelin E, Aleman C. Eur Polym J 2008; 44: 13231330.

[29] Oliver R, Munoz A, Ocampo C, Aleman C, Estrany F. Chem Phys 2006; 328: 299-306.

[30] Aradilla D, Estrany F, Armelin E, Alemán C. Thin Solid Films 2012; 520: 4402-4409.

[31] Van Hoang H, Holze R. Chem Mater 2006; 18(7): 1976-1980. 
[32] Xia Y, Li T, Ma C, Gao C, Chen J. RSC Adv 2014; 4: 20516-20520.

[33] Letaïef S, Aranda P, Fernández-Saavedra R, Margeson JC, Detelliera C, Ruiz-Hitzky E. J Mater Chem 2008; 18: 2227-2233.

[34] Aranda P, Darder M, Fernandez-Saavedra R, Lopez-Blanco M, Ruiz-Hitzky E. Thin Solid Films 2006; 495: 104-112.

[35] Rajesh, T. Ahuja and D. Kumar, Sens. Actuators. B 2009; 136: 275-286.

[36] Rajapakse RMG, Higgins S, Velauthamurthy K, Bandara HMN, Wijeratne S, Rajapakse RMMY. J Compos Mater 2010; 45: 597-608.

[37] Han Y, Lu Y. J Appl Polym Sci 2009; 11: 2400-2407.

[38] Aradilla D, Estrany F, Azambuja DS, Casas MT, Puiggalí J, Ferreira CA, Aleman C. Eur Polym J 2010; 46: 977- 983.

[39] Bae WJ, Kim KH, Park YH, Jo WH. Macromolecules 2004; 37: 9850-9854.

[40] Sanchez-Jimenez M, Aleman C, Estrany F. Polym Eng \& Sci 2014; 54: 2122-2131.

[41] Aradilla D, Estrany F, Armelin E, Alemán C. Thin Solid Films 2010; 518: 4203-4210.

[42] Aradilla D, Estrany F, Alemán C. J Appl Polym Sci 2011; 121:1982-1991.

[43] Aradilla D, Estrany F, Alemán C. Org Electron 2013; 14: 131-142

[44] Murugan AV, Viswanath AK, Gampet G, Gopinath CS, Vijayamohanan K. Appl Phys Lett 2005; 87: 243511.

[45] Sharma R, K. Zhai L. Electrochim Acta 2009; 54: 7148-7155.

[46] Sen PM, De M. Electrochim. Acta, 2010; 55: 4677-4684.

[47] Babakhani B, Ivey DG. Electrochim. Acta, 2010; 55: 4014-4024.

[48] Chen L, Yuan C, Gao B, Chen S, Zhang X. J Solid State Electrochem 2009; 13: 19251933.

[49] Murugan AV, Viswanath AK, Gopinath, CS, Vijayamohanan K. J Appl Phys 2006; 100: 074319. 
Table 1. Specific capacitances $\left(\mathrm{SC}\right.$, in $\mathrm{F} \cdot \mathrm{g}^{-1}$ ) of 3-layered films obtained using $\theta_{P N M P y}=$ $600 \mathrm{~s}$ and different $\theta_{P E D O T}$ values. SC were determined for as prepared fresh samples and for samples submitted to 100 consecutive oxidation-reduction cycles.

\begin{tabular}{cccc}
\hline Film & $\begin{array}{c}\theta_{P E D O T} \\
(\mathrm{~s})\end{array}$ & $\begin{array}{c}\text { SC } \\
\text { (fresh samples) }\end{array}$ & $\begin{array}{c}\text { SC } \\
\text { (100 redox cycles) }\end{array}$ \\
\hline PEDOT/PNMPy/PEDOT & 10 & 445 & 82 \\
& 50 & 172 & 63 \\
& 300 & 54 & 34 \\
PEDOT/PNMPy-MMT/PEDOT & 10 & 333 & 103 \\
& 50 & 121 & 78 \\
& 300 & 75 & 58 \\
\hline
\end{tabular}




\section{CAPTIONS TO FIGURES}

Figure 1. Scheme of the synthetic procedure used to prepare PEDOT/PNMPyMMT/PEDOT.

Figure 2. TEM micrograph of PNMPy-MMT generated using $\theta_{P N M P y}=180 \mathrm{~s}$ and deposited onto a micrometric PEDOT film.

Figure 3. AFM images of: (a) PNMPy and (b) PNMPy-MMT films generated using $\theta_{P N M P y}=180 \mathrm{~s}$ and deposited onto a micrometric PEDOT film $\left(\theta_{P E D O T}=300 \mathrm{~s}\right)$; (c) PNMPy and (d) PNMPy-MMT films generated using $\theta_{P N M P y}=600 \mathrm{~s}$ and deposited onto a micrometric PEDOT film $\left(\theta_{\text {PEDOT }}=300 \mathrm{~s}\right.$ ); (e) PNMPy and (f) PNMPy-MMT films generated using $\theta_{P N M P y}=600 \mathrm{~s}$ and deposited onto a nanometric PEDOT film of $\theta_{P E D O T}=50 \mathrm{~s} .2 \mathrm{D}$ (left) and 3D (right) topographic images.

Figure 4. 3D AFM topographic images of the internal PEDOT layer prepared using $\theta_{\text {PEDOT }}=(\mathrm{a}) 300 \mathrm{~s}$ and (b) $50 \mathrm{~s}$.

Figure 5. SEM micrographs of (a and c) PNMPy and (b and d) PNMPy-MMT $\left(\theta_{P N M P y}=\right.$ $600 \mathrm{~s}$ ) deposited onto an internal (a and b) nanometric and (c and d) micrometric PEDOT layer $\left(\theta_{P E D O T}=10\right.$ and $300 \mathrm{~s}$, respectively). (e) SEM micrograph of the internal PEDOT layer prepared using $\theta_{P E D O T}=300 \mathrm{~s}$.

Figure 6. Cyclic voltammograms for representative systems. (a) PEDOT/PNMPyMMT/PEDOT and (b) PEDOT/PNMPy/PEDOT prepared using $\theta_{P E D O T}=300 \mathrm{~s}$ and $\theta_{P N M P y}=$ 600 s: first control voltammogram (black solid line) and voltammogram after 100 consecutive oxidation-reduction cycles (grey dashed line). (c) PEDOT/PNMPy-MMT/PEDOT and (d) PEDOT/PNMPy/PEDOT prepared using $\theta_{P E D O T}=50 \mathrm{~s}$ and $\theta_{P N M P y}=600 \mathrm{~s}$ : first control voltammogram (black solid line) and voltammogram after 10 consecutive oxidation-reduction cycles (grey dashed line). 
Figure 7. Electroactivity difference ( $\Delta$-Electroactivity) between PEDOT/PNMPyMMT/PEDOT and PEDOT/PNMPy/PEDOT films prepared using the same $\theta_{\text {PEDOT }}(10,40$ or $100 \mathrm{~s}$ ) and $\theta_{P N M P Y}=600 \mathrm{~s}$ that initially showed an anomalous behavior (i.e. the electoactivity of the latter was higher than that of the former), against the number of consecutive oxidationreduction cycles. After only 6-7 redox cycles, the electroactivity order is reversed.

Figure 8. Variation of the loss of electroactivity (LEA, Eqn 1) with respect to the number of consecutive oxidation-reduction cycles for (a) PEDOT/PNMPy/PEDOT and (b) PEDOT/PNMPy-MMT/PEDOT films prepared using $\theta_{\text {PEDOT }}$ ranging from 10 to $300 \mathrm{~s}$ and $\theta_{P N M P y}=600 \mathrm{~s}$.

Figure 9. Specific capacitance (SC, Eqn 2) of PEDOT/PNMPy/PEDOT and PEDOT/PNMPy-MMT/PEDOT films $\left(\theta_{P N M P y}=600 \mathrm{~s}\right)$ as a function of $\theta_{\text {PEDOT }}$.

Figure 10. Chronopotentiograms recorded for the oxidation of PEDOT/PNMPy/PEDOT and PEDOT/PNMPy-MMT/PEDOT films prepared using $\theta_{P E D O T}=10,50$ or $100 \mathrm{~s}$ and $\theta_{P N M P y}=600 \mathrm{~s}$ in acetonitrile with $0.1 \mathrm{M} \mathrm{LiClO}_{4}$. Applied current density: $2 \mathrm{~mA} / \mathrm{cm}^{2}$. Temperature: $25^{\circ} \mathrm{C}$. 

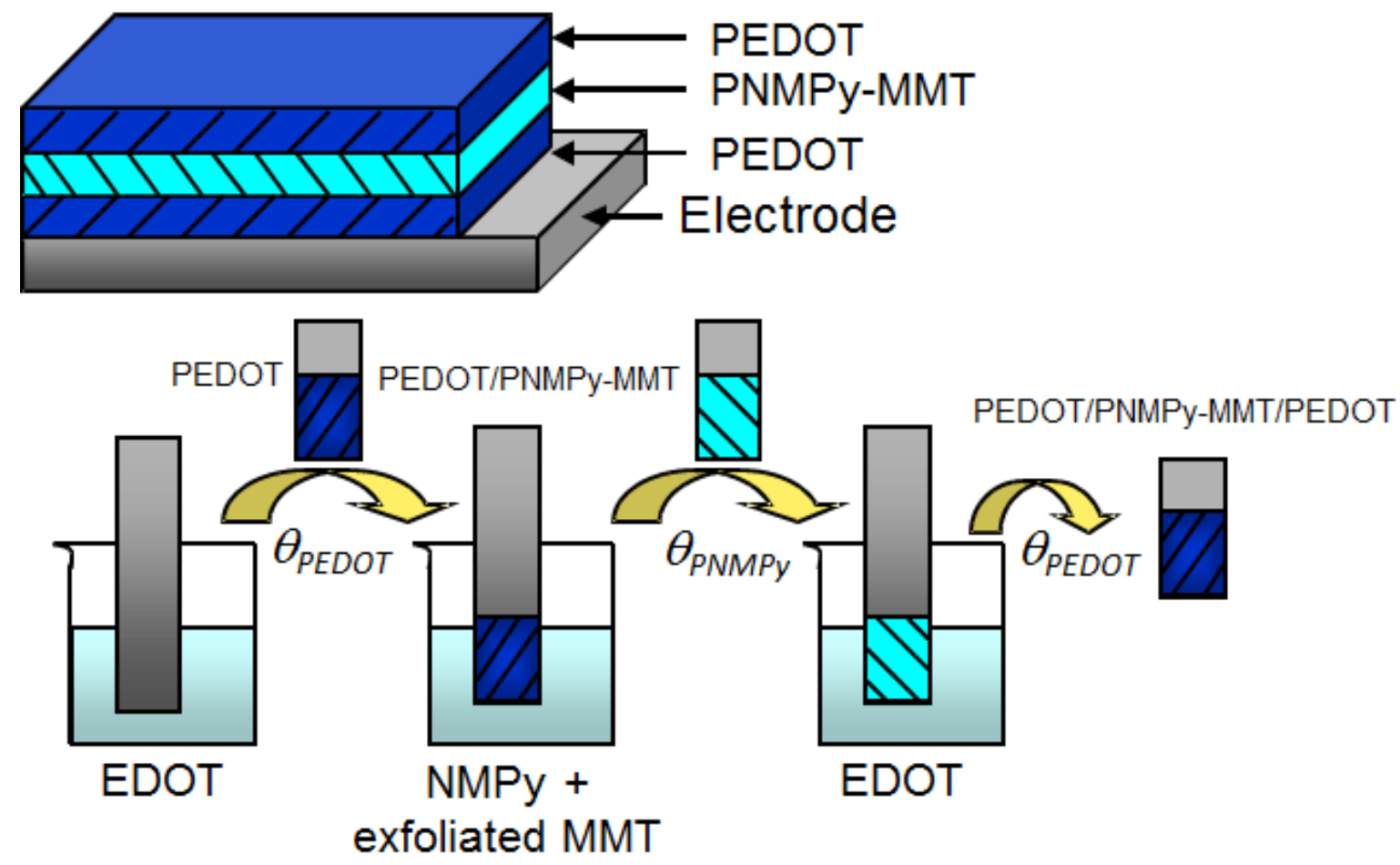

Figure 1 
Figure 2 

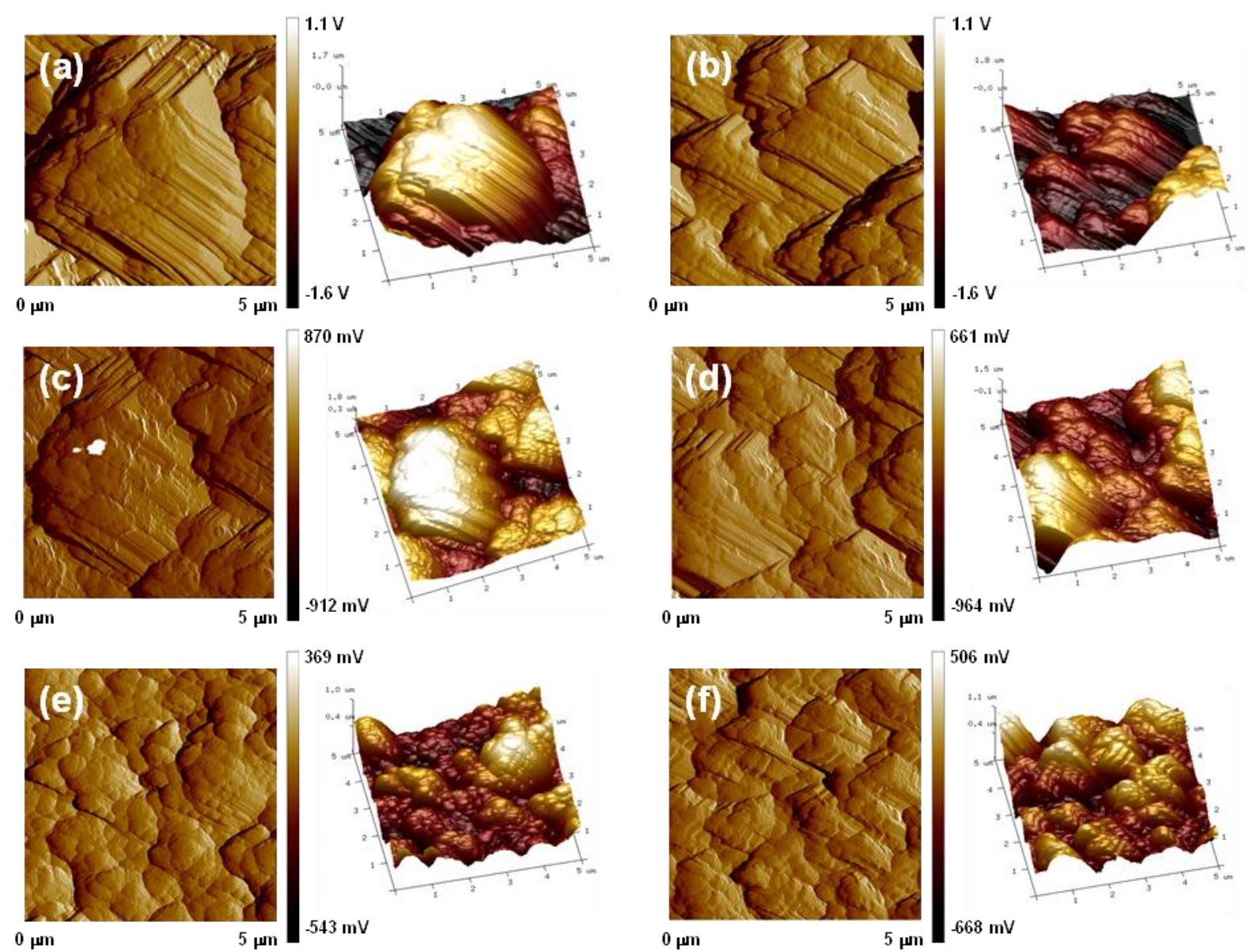

Figure 3 
(a)

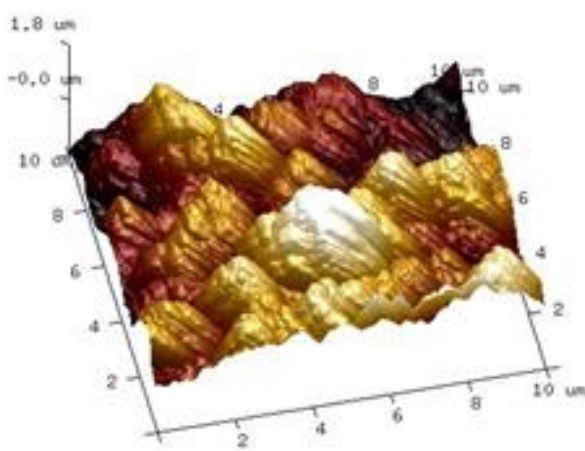

(b)

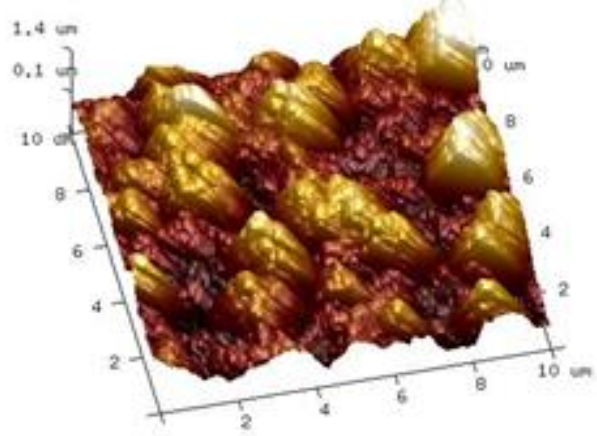

Figure 4 

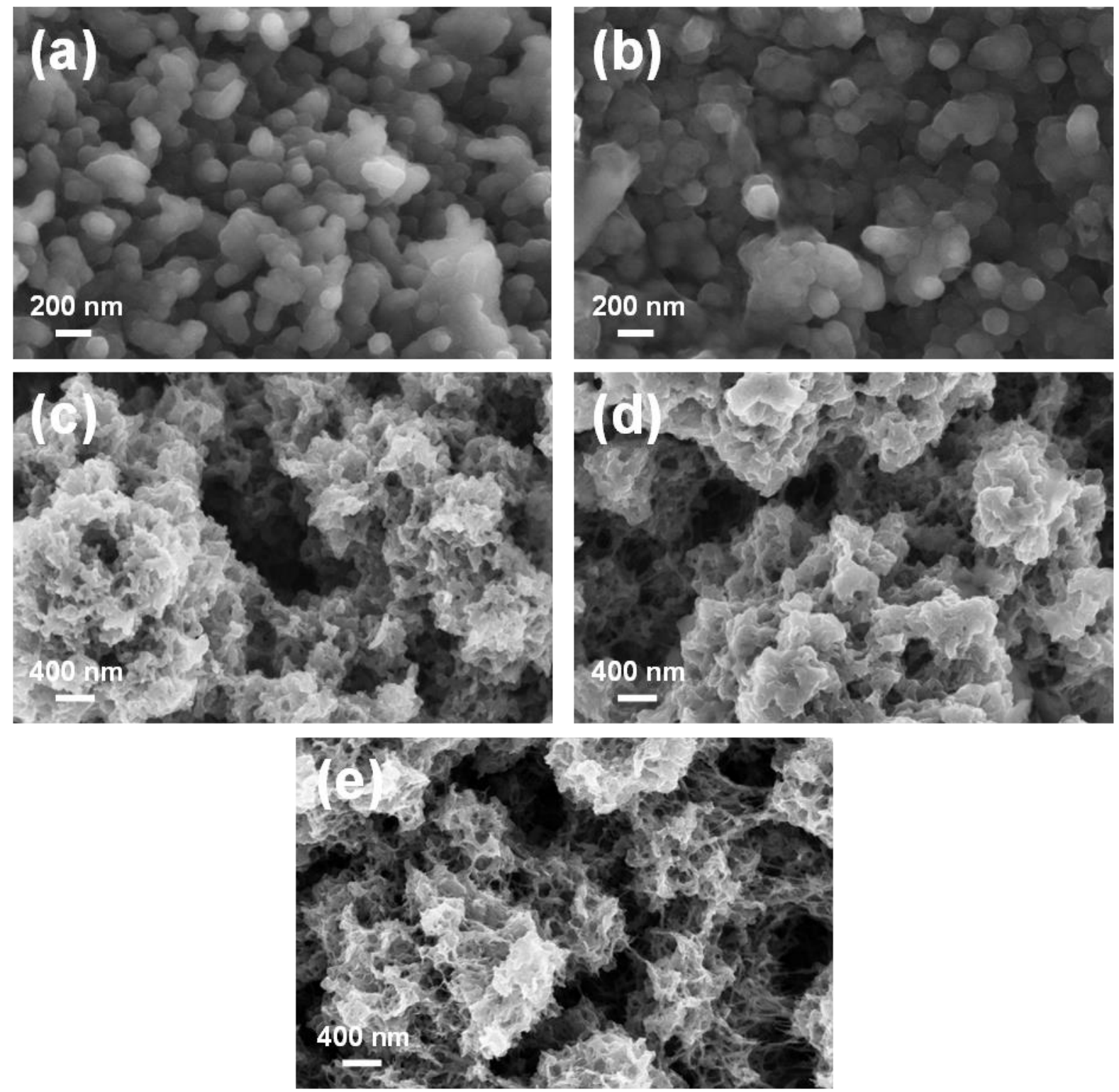

Figure 5 

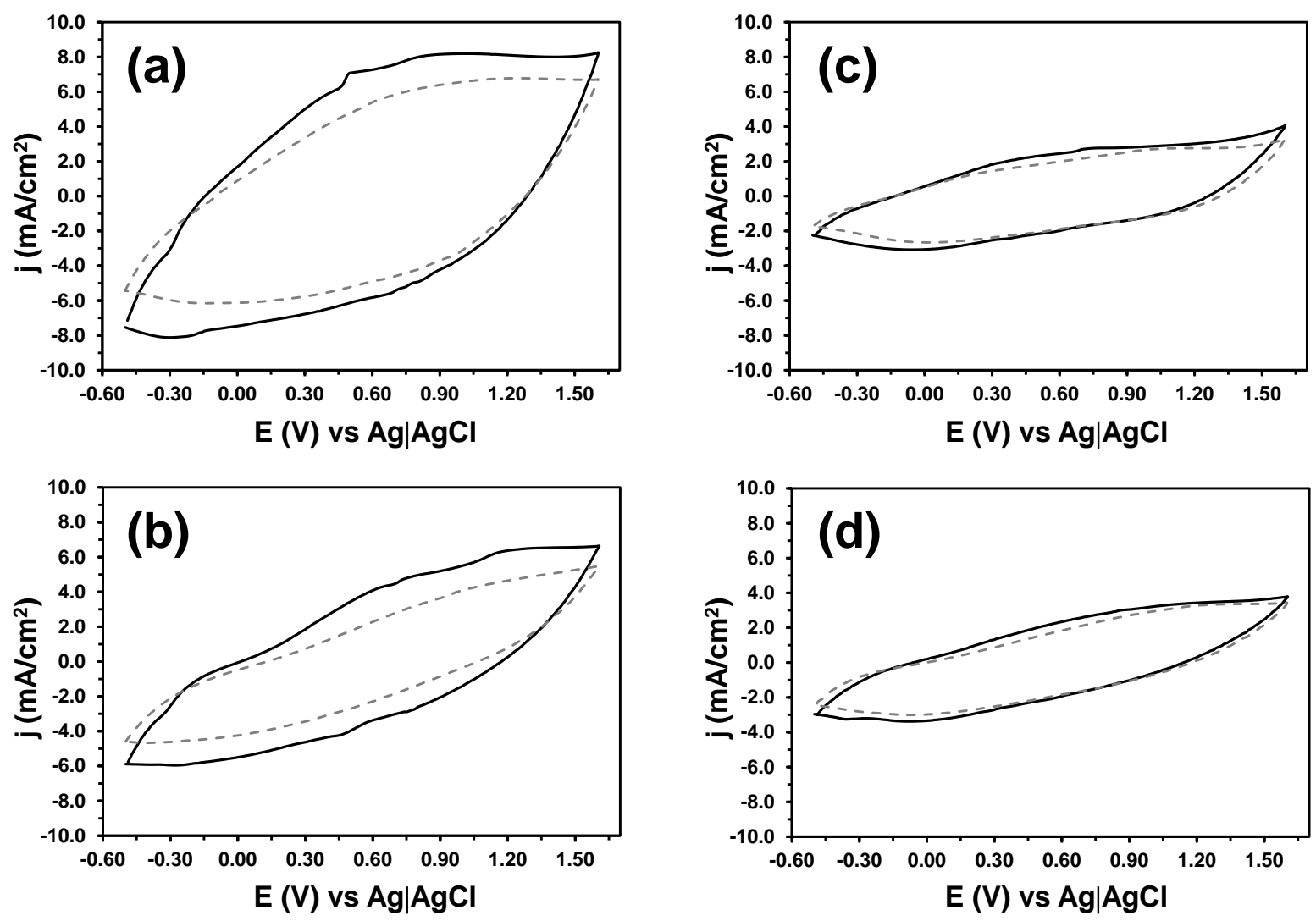

Figure 6 


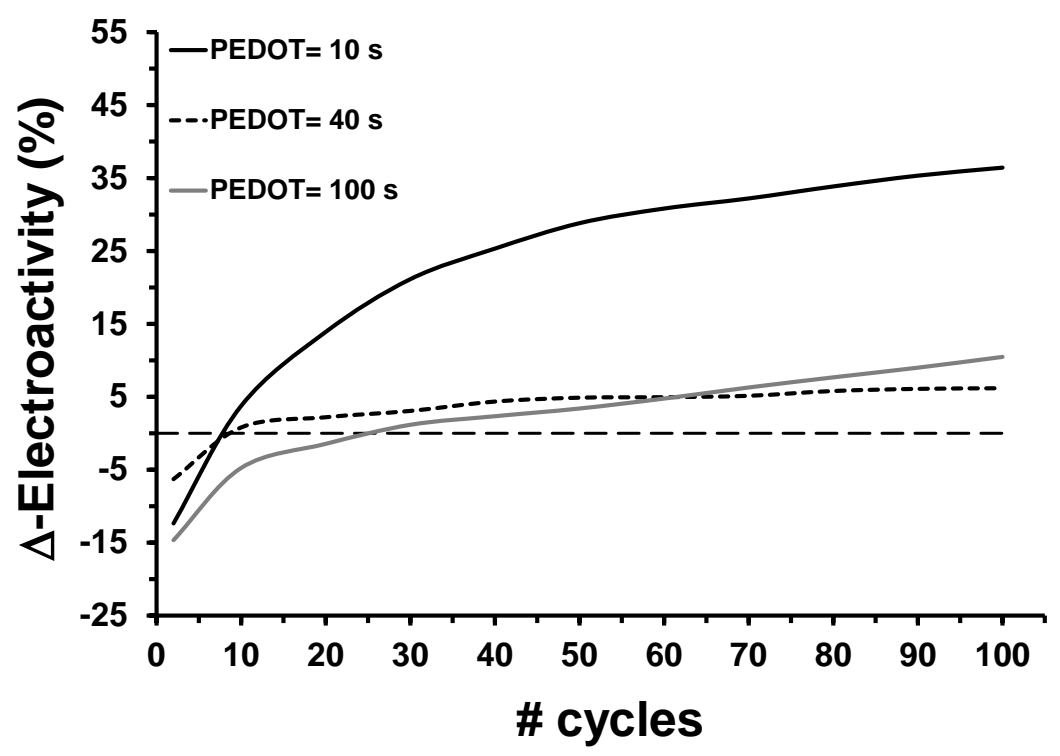

Figure 7 

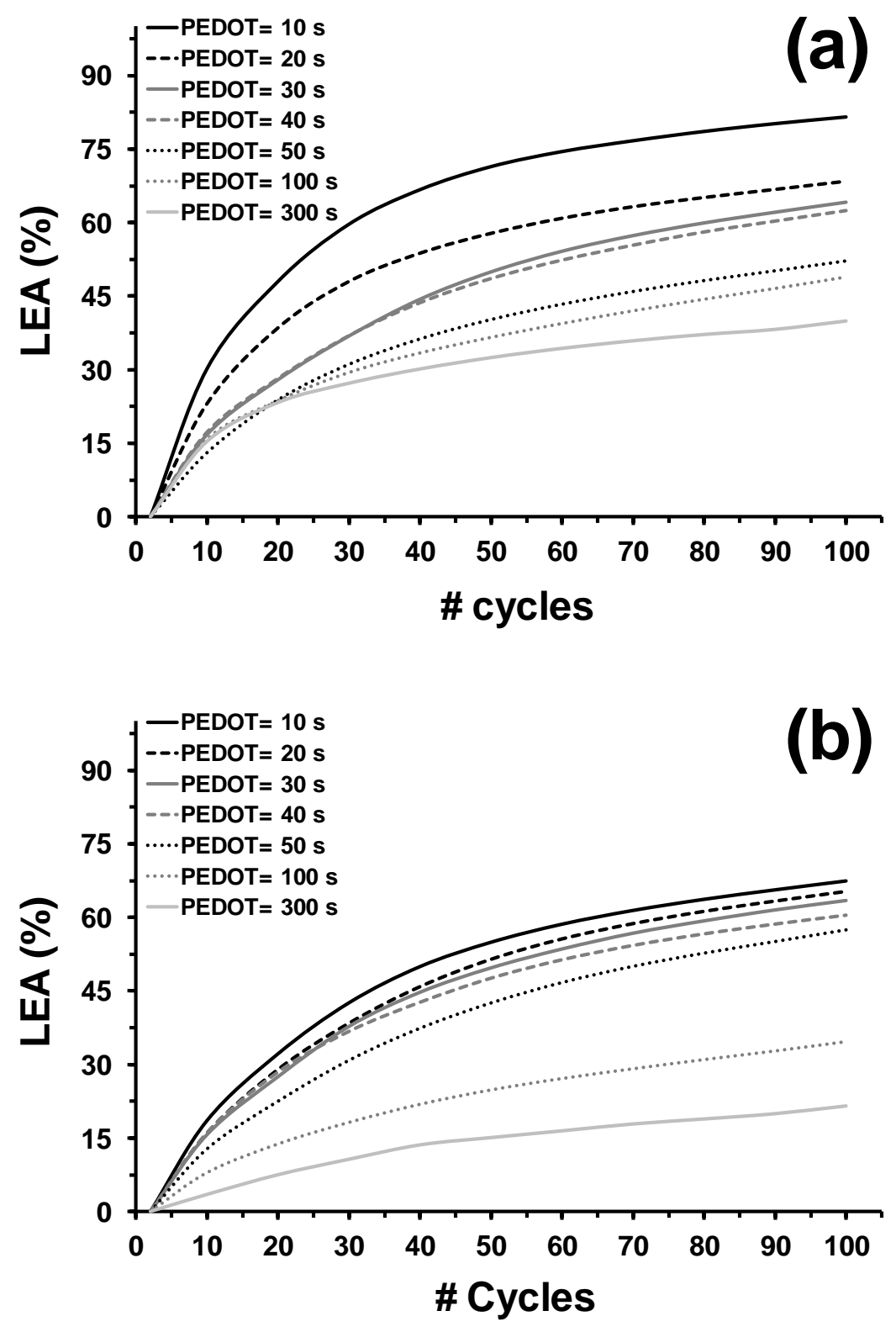

Figure 8 


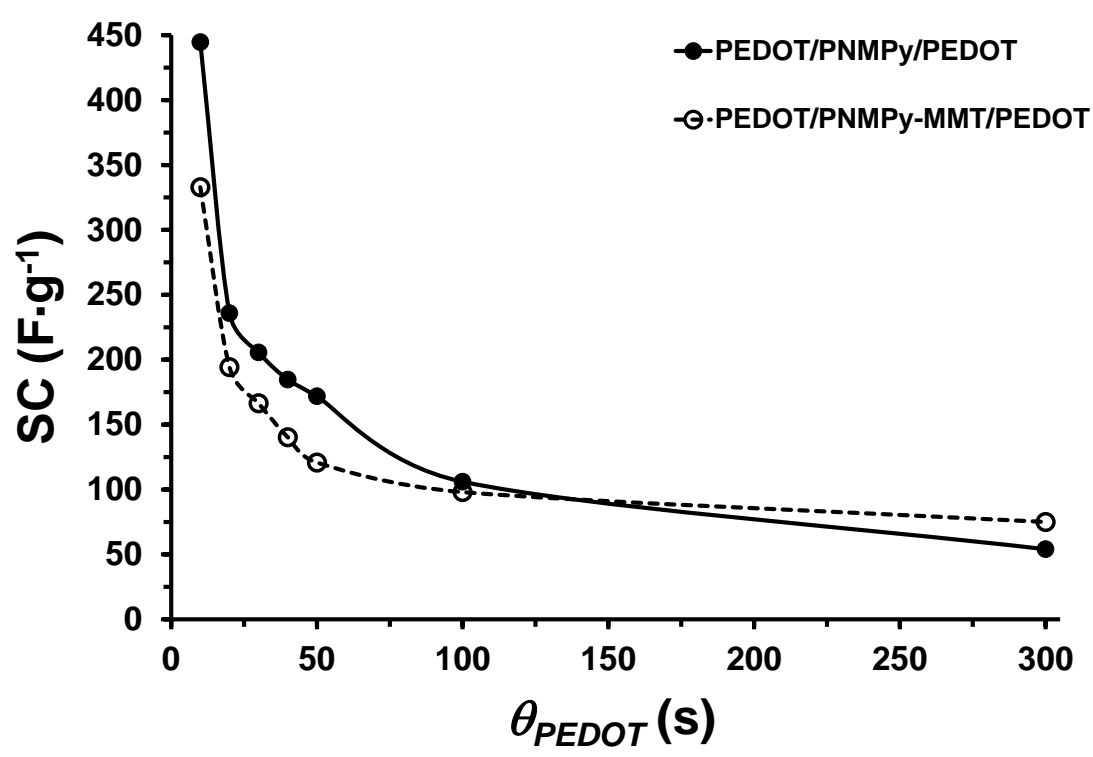

Figure 9 


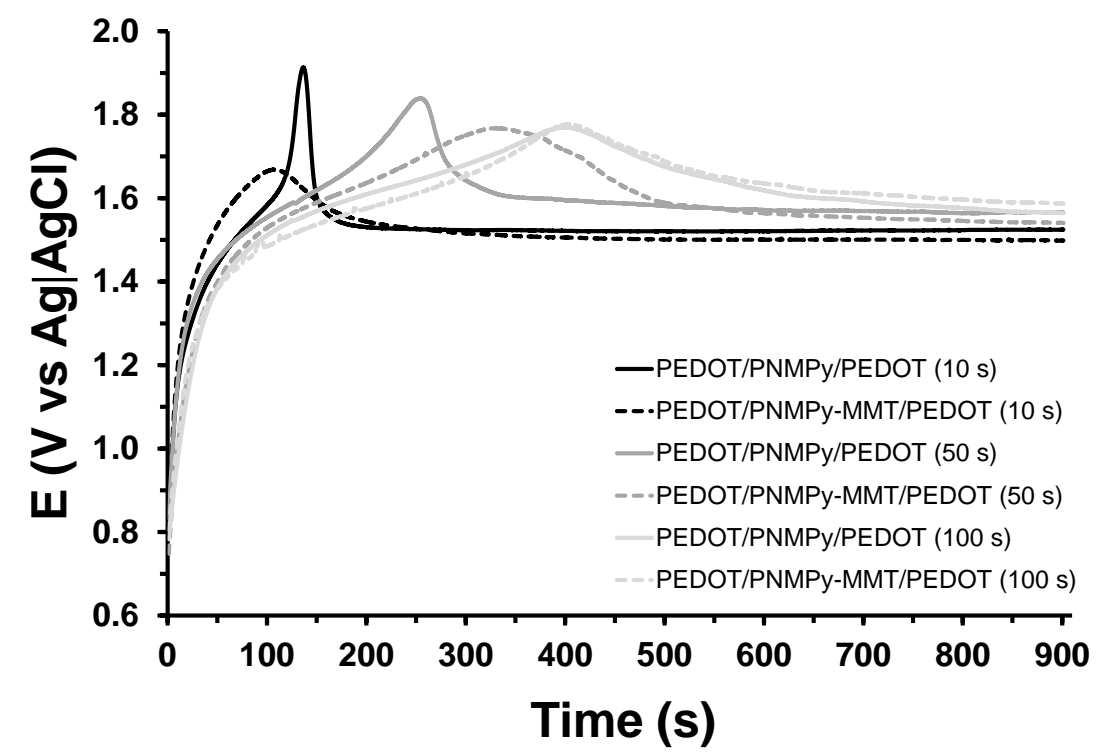

Figure 10 